\title{
Young stellar clusters in the Rosette molecular cloud
}

\section{Arguments against triggered star formation $\star, \star \star$}

\author{
L. Cambrésy ${ }^{1}$, G. Marton ${ }^{2}$, O. Feher ${ }^{3}$, L. V. Tóth ${ }^{3}$, and N. Schneider ${ }^{4}$ \\ ${ }^{1}$ Observatoire astronomique de Strasbourg, Université de Strasbourg, CNRS, UMR 7550, 11 rue de l'Université, 67000 Strasbourg, \\ France \\ e-mail: cambresy@astro.unistra.fr \\ 2 Konkoly Observatory, Research Centre for Astronomy and Earth Sciences, Hungarian Academy of Sciences, Konkoly Thege 15-17, \\ 1121 Budapest, Hungary \\ 3 Eötvös Loránd University, Department of Astronomy, Pázmány Péter sétány 1/A, 1117 Budapest, Hungary \\ ${ }^{4}$ Laboratoire d'Astrophysique de Bordeaux, CNRS/INSU, Université de Bordeaux, BP 89, 33271 Floirac Cedex, France
}

Received 5 February 2013 / Accepted 15 July 2013

\section{ABSTRACT}

\begin{abstract}
Aims. We focus on characterizing the young stellar population in the Rosette complex to improve our understanding of the processes that regulate the star formation in this region.

Methods. We propose an original method that relies on the joint analysis of the star color and density in the near-infrared. It leads to mapping the molecular cloud spatial distribution and detecting the embedded clusters with their characterization in terms of member number and age estimation.

Results. We have identified 13 clusters, 2 of which are new discoveries, and we estimate that the total number of young stellar objects in the Rosette ranges between 4000 and 8000 members. We find that the age distribution of the young clusters is not consistent with a general triggered scenario for the star formation in this molecular cloud.

Conclusions. This study proves that the Rosette complex evolution is not governed by the influence of its OB star population. It suggests that the simple morphological appearance of an active region is not sufficient to conclude much about the triggering role in the star formation process. Our method of constraining the cluster properties using UKIDSS and WISE data has proven efficient, and studies of other regions of the Galactic plane would definitely benefit from this approach.
\end{abstract}

Key words. stars: pre-main sequence - dust, extinction - infrared: general - ISM: individual objects: Rosette molecular cloud

\section{Introduction}

The Rosette complex is a well studied region of the Galactic plane that presents the apparent characteristics of a triggered star forming region. This is, however, still being debated since no strong evidence corroborates this statement. It is composed of an OB star cluster that illuminates the famous optical nebula and interacts with a molecular cloud that contains several star clusters. The review by Román-Zúñiga \& Lada (2008) gives a detailed introduction to this complex. The well-known region has been observed from the radio to the X-ray wavelengths. The analysis of the star population in the Rosette molecular cloud (RMC) suggests a sequential formation (Román-Zúñiga et al. 2008), with the more recent clusters farther from NGC 2244.

The mechanisms occurring in triggered star forming regions (see the review by Elmegreen 1998) are indeed fully compatible with the geometry of the Rosette complex, which does appear to be a perfect candidate; however, the studies based on the interstellar medium rather than the stars state otherwise. Celnik (1985) observed the radio recombination line in the Rosette HII and showed the nebula is ionization bound, which suggests that

\footnotetext{
* Appendix A is available in electronic form at http://www . aanda.org

$\star \star$ Full Table 4 is only available at the CDS via anonymous ftp to cdsarc.u-strasbg.fr (130.79.128.5) or via http://cdsarc.u-strasbg.fr/viz-bin/qcat?]/A+A/557/A29
}

the clusters outside the ionization front are too far from the central $\mathrm{O}$ stars for efficient triggering to take place. Heyer et al. (2006) confirm this claim after studying the turbulent fragmentation in the Rosette. They find that the velocity structures due to the expansion of ionized gas have not yet propagated through the cloud to significantly modify its dynamics. Schneider et al. (2012) reached the same conclusion using Herschel observations to reveal the filamentary structure of the RMC. They find that clusters lie at the junction of filaments as predicted by turbulence simulations, including radiative feedback (Dale \& Bonnell 2011). Poulton et al. (2008) obtained Spitzer IRAC and MIPS data and also expressed doubt about the preponderance of triggering in the Rosette. Their conclusion is essentially based on the fact that some clusters are outside the ionization front and that they did not observed any overdensity of very young objects at the front position itself. A more local influence has been investigated by Balog et al. (2007). They show that the OB stars in NGC 2244 contribute to the photo-evaporation of a low-mass star circumstellar disk when they are located within $0.5 \mathrm{pc}$.

In this paper we perform a large scale study of the Rosette complex by analyzing the interstellar medium distribution and star cluster properties. The datasets we used are presented in Sect. 2. Section 3 describes the extinction mapping of the RMC that allows an unbiased cluster characterization. Section 4 focuses on the analysis of the clusters leading to their detection and the estimation of their total number of members. The individual 
identification of members followed by an age study is presented in Sect. 5. We summarize the results and conclude in Sect. 6. Finally, an appendix provides the reader with a comparison between the column density derived from background source reddening and from dust emission in the submillimeter observed with Herschel.

\section{Datasets}

The near-infrared $J H K_{\mathrm{S}}$ data were obtained from the UKIDSS (Lawrence et al. 2007) Data Release 8 (DR8) of the Galactic Plane Survey for a region defined by the coordinate range $l \in$ [204.5, 209.0] and $b \in[-3.5,-0.5]$. UKIDSS uses the UKIRT Wide Field Camera (WFCAM; Casali et al. 2007). The photometric system is described in Hewett et al. (2006), and the calibration is described in Hodgkin et al. (2009). The pipeline processing and science archive are described in Hambly et al. (2008). The $K_{\mathrm{s}}$ image located at $(l, b)=(206.25,-2.85)$ was not selected for the DR8 owing its lower quality. The missing sources were taken from the DR7 source list. After removing all duplicate sources in UKIDSS, the sample contains 1.4 million objects with a completeness limit of 17.8 mag at $K_{\mathrm{s}}$. To avoid instrumental saturation issues, the photometry of sources brighter than 12 mag has been replaced by 2 MASS magnitudes at $J, H$, and $K_{\mathrm{S}}$ (Skrutskie et al. 2006). We also used data from the Widefield Infrared Survey Explorer (WISE, Wright et al. 2010) for young stellar object (YSO) identification. It provided us with $2.4 \times 10^{5}$ sources within our field of interest with the photometry from 3.4 to $22 \mu \mathrm{m}$.

\section{Extinction mapping of the RMC}

The first step in our study consists in mapping the extinction in the whole molecular cloud. This is required because the dust extinction reddens stars and decreases their apparent density. Several approaches are possible, such as using $\mathrm{CO}$ or dust emission. Carpenter (2000) used CO observations to trace the extinction before performing a density analysis to extract young clusters, but CO observations are subject to detection threshold, photodissociation, depletion, and line saturation. The submillimeter dust emission mapped by Herschel is another possibility for tracing the dust distribution (Schneider et al. 2010). One drawback is the assumption of uniform dust properties, e.g. a constant emissivity for the whole cloud and a single efficient temperature along each line of sight.

The technique we chose for generating the extinction map is described extensively in Cambrésy et al. (2002, 2011). We only provide the reader with the outlines of the method here. Basically, it is an adaptive process where extinction is estimated from the median color of the three nearest neighbors. The median filters the outliers. The final map is obtained by a convolution by an adaptive kernel that produces an extinction map with uniform spatial resolution. This is different from directly using a regular grid from the start since it minimizes the nonlinear bias caused by the spatial distribution of the stars, under-represented at high extinction.

In this section, we restrict our source list to the objects simultaneously detected at $H$ and $K_{\mathrm{s}}$, with an uncertainty smaller than $0.15 \mathrm{mag}$ and $K_{\mathrm{S}}<17.8 \mathrm{mag}$. Since the RMC is located in the Galactic plane at $1.6 \mathrm{kpc}$, foreground stars become the dominant population in the cloud's darkest regions and must be removed. The degeneracy between intrinsically red foreground stars and blue background stars prevents their individual classification through a color cut-off. However this degeneracy is raised

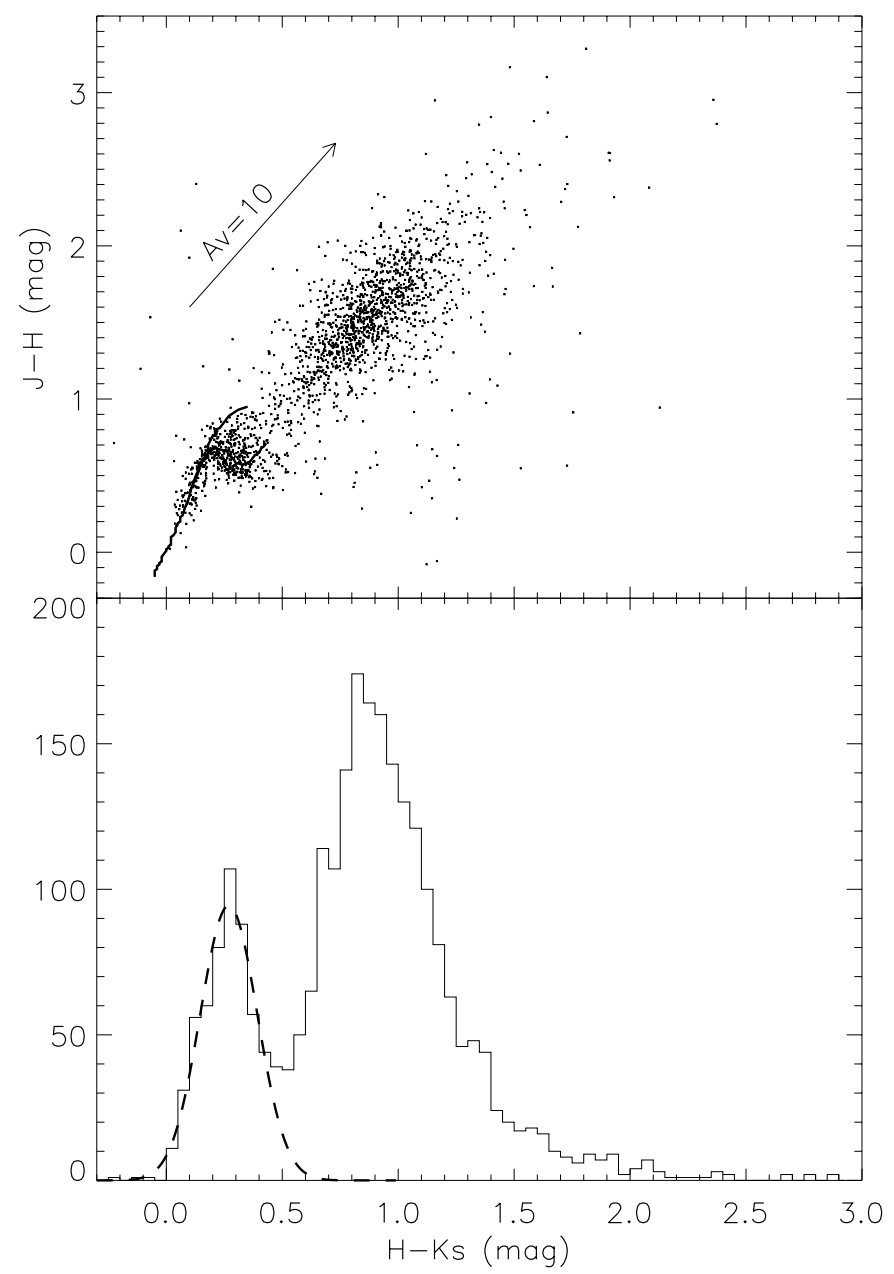

Fig. 1. Top: color-color diagram for sources in the regions where $A_{V}>$ 10 mag, excluding the cluster areas. The two groups correspond to foreground and background stars. The arrow represents the reddening vector for 10 mag of visual extinction. Bottom: color histogram for the same sources. The dashed line is a Gaussian fit of the bluer peak, yielding a foreground star density of $2.2 \pm 0.1 \mathrm{arcmin}^{-2}$.

at high extinction allowing foreground stars to be identified and their density estimated. Since the foreground object surface density is uniform over a reasonably large area, an efficient way to correct from this contamination is to subtract the estimated star density over the whole field. We found a foreground star surface density of $2.2 \pm 0.1 \mathrm{arcmin}^{-2}$, i.e., $7920 \pm 340 \mathrm{deg}^{-2}$ (see Fig. 1). In the densest part of the cloud, it almost corresponds to the total star density, which indicates that very few background objects are actually detected for these lines of sight. Once the foreground surface density is measured, we still need to decide which stars to remove. We select the bluer stars, which are the more likely to be foreground. This truly removes the foreground sources at high extinction. At lower extinction, the selected stars are not necessarily foreground because of the degeneracy issue mentioned above. It actually does not matter because the total median density over the whole field is $16 \mathrm{arcmin}^{-2}$, which means less than $14 \%$ of the star are foreground. Removing the bluer ones marginally affects the median color estimation. At this point, about $10^{5}$ stars are removed from our source list. It worth noting that this method has been validated for the more complex case of the Trifid molecular cloud (Cambrésy et al. 2011), which is closer to the Galactic center direction with $(l, b) \approx(7,0)$ deg and 


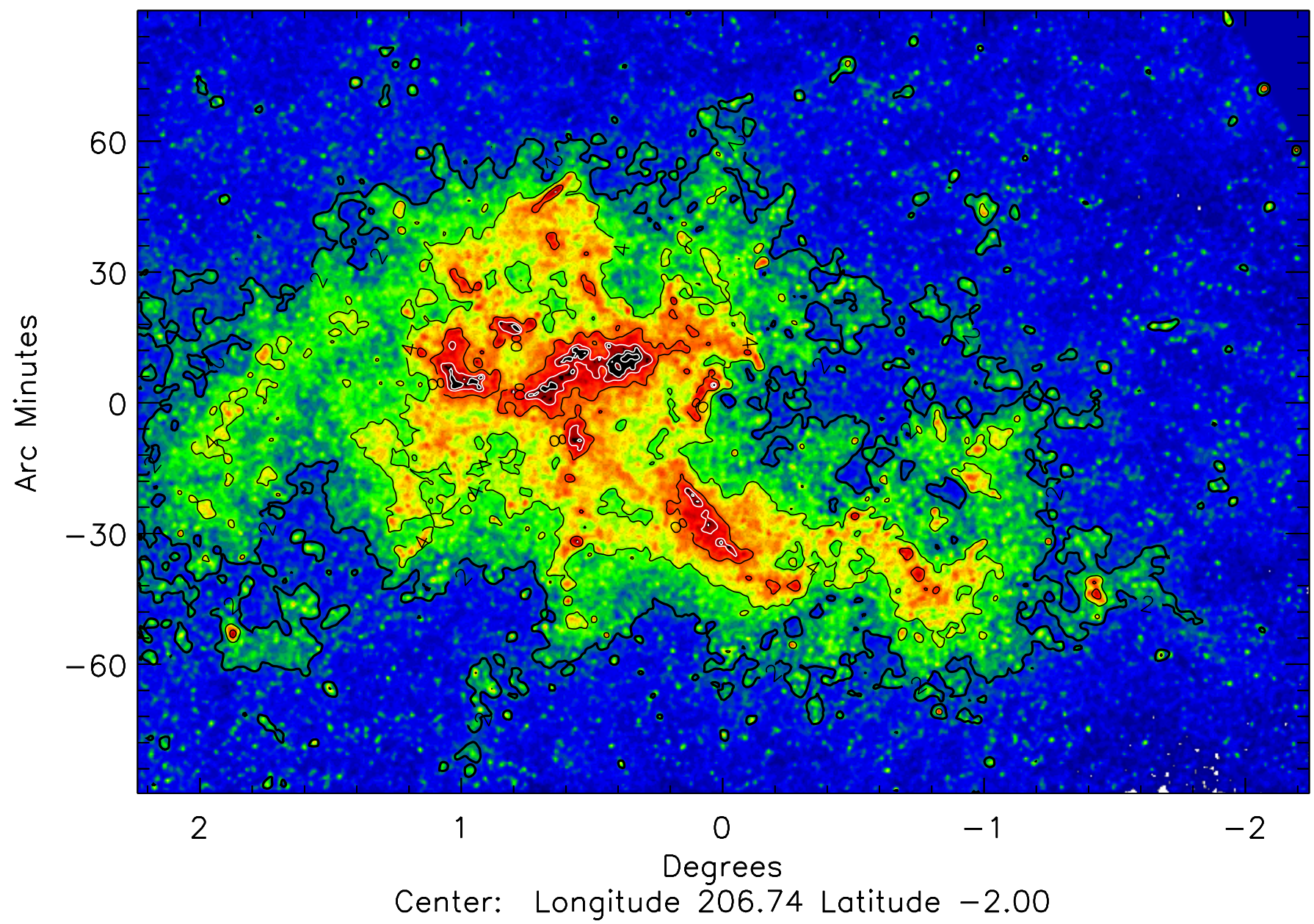

Fig. 2. Extinction map of the Rosette molecular cloud at $1^{\prime}$ resolution. Contour levels are for visual extinctions of 2, 4, 8 mag in black and 12, 16, 24 mag in white. The maximum extinction peak reaches $A_{V}=38$ mag.

at a larger distance of $2.7 \mathrm{kpc}$. A variant but similar approach is proposed by Kainulainen et al. (2011) for infrared dark clouds.

The final extinction map at a spatial resolution of $1^{\prime}$ is presented in Fig. 2. It is obtained from $H-K_{\mathrm{s}}$ color excess using the Rieke \& Lebofsky (1985) extinction law, which is consistent with our data. The extinction law variations can be critical in optical wavelength and beyond $3.5 \mu \mathrm{m}$, but few variations are expected between 1 and $2 \mu \mathrm{m}$. The extinction is set to zero on the edge of the cloud. No diffuse extinction along the line of sight has been added so that the extinction map truly represents the RMC column density. Assuming a distance of $1.6 \mathrm{kpc}$ and the gas-to-dust ratio $N_{\mathrm{H}} / A_{V}=1.87 \times 10^{21} \mathrm{~cm}^{-2} \mathrm{mag}^{-1}$ (Savage $\&$ Mathis 1979), we can derive the mass of the cloud. The cumulative mass distribution expressed as the mass enclosed in a given extinction isocontour (see Fig. 3) follows this powerlaw for $A_{V}<20$ mag:

$$
M\left(A_{V}\right)=M(0) \times 10^{-\alpha A_{V}}
$$

where the total mass of the cloud $M(0)=4.3 \times 10^{5} M_{\odot}$ and the index $\alpha=0.122 \pm 0.005$. For comparison the total mass of the Orion molecular cloud is about $3 \times 10^{5} M_{\odot}$. The relation becomes flatter at extinctions higher than 20 mag reminding the observed behavior in the Trifid molecular cloud by Cambrésy et al. (2011). However, as only $14 \mathrm{arcmin}^{2}$ reach this level in the $\mathrm{RMC}$ we prefer not to elaborate about this aspect. Independent mass estimations are available in the literature. Williams et al. (1995) proposed a mass ranging from $1.1 \times 10^{5} M_{\odot}$ to $2.2 \times 10^{5} M_{\odot}$ from CO observations. This is significantly less than our value, but mass estimations from $\mathrm{CO}$ suffer several issues related to photodissociation, threshold detection, and line opacity. Schneider et al. (2010) obtained $10^{5} M_{\odot}$ from dust emission using Herschel data for a smaller surface area. Our mass estimate drops to $1.4 \times 10^{5} M_{\odot}$ if we restrict the initial $13 \mathrm{deg}^{2}$ of our map to the $1.8 \mathrm{deg}^{2}$ of the Herschel map, making both estimations consistent.

Besides reddening the sources, the extinction produces variations in the star density. For instance, a visual extinction of 5 mag implies the disappearance of one third of the background sources at $K_{\mathrm{s}}$. The extinction map is essential to correct the stellar density before performing any clustering analysis, as explained in Sect. 4.1.1.

\section{Cluster identification}

\subsection{Preparation}

A quick-look surface density analysis is obtained through a $1^{\prime}$ sampling star count of the $K_{\mathrm{s}}$ sources. Instead of a map we actually built a datacube where each plane is a density map for sources up to a magnitude limit ranging from 11 to 20 mag in steps of $0.1 \mathrm{mag}$. The main star clusters are detectable by eye when browsing the datacube from the bright to faint stars. This datacube permits the magnitude distribution of the cluster members to be probed using the differential stellar density, defined as 


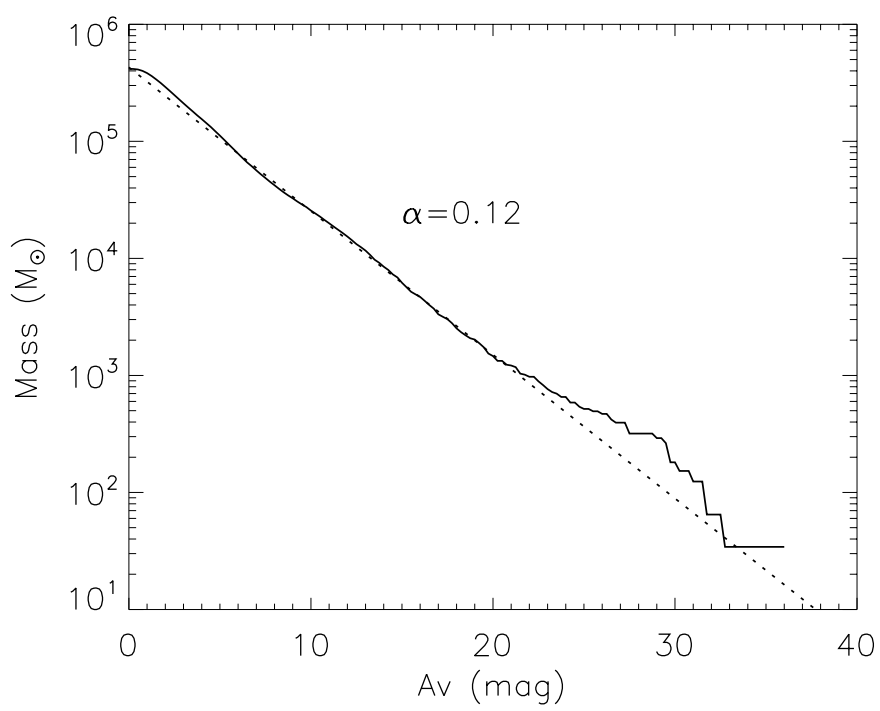

Fig. 3. Cumulative mass distribution for the Rosette molecular cloud. The dashed line follows the powerlaw defined by Eq. (1).

the density of stars with $K_{\mathrm{s}} \in[m-1, m]$. The differential stellar density ratio between pixels covering the cluster areas (on) and pixels excluding the cluster areas (off) increases with the magnitude up to about $K_{\mathrm{S}} \approx 14$ mag and then decreases when counting fainter sources (Fig. 4a). Figure 4 b presents the two differential densities for the cluster on and off positions. The plot indicates that the clusters essentially contain stars brighter than 17 mag at $K_{\mathrm{s}}$, which is a direct consequence of their distance and age population. These plots teach us that the signal-to-noise ratio in the clustering analysis will be enhanced by restricting the sample to sources with $K_{\mathrm{s}} \in[12.5,15.5] \mathrm{mag}$. This range is a good compromise with a high density contrast and a sample that still includes about $65 \%$ of the cluster members. Finally, the question of the contamination by galaxies must be addressed. It appears to be negligible for this magnitude limit with an estimated density of $\sim 0.1 \operatorname{arcmin}^{-2}$, about 50 times less than the star density as shown Fig. 4b.

\subsubsection{Density map}

The historical and trivial technique for mapping the stellar surface density is to count stars within each cell of a regular grid. Such a density estimator is actually the classical 2D histogram for which cell size has to be defined in advance for the whole field, and the grid initial position matters. The concept of an adaptive grid used for the extinction mapping yields an improved density estimator. It consists of setting the number of sources per cell rather than the grid size. The local density, $D$, is then derived from the distance, $r$, of the $N$ th nearest neighbor by

$D=\frac{N-1}{\pi r^{2}}$

Casertano \& Hut (1985) demonstrated that counting the $N$ th star produces a bias in the density estimation. This is why $N-1$ is preferred in the Eq. (2) numerator. There are other statistical methods for estimating the density (see for example Silverman 1986), but the nearest-neighbor method has proven to be efficient for stellar distributions and is indeed widely used in the Rosette complex (Román-Zúñiga et al. 2008; Poulton et al. 2008; Wang et al. 2009). By using $N=20$ for stars with $K_{\mathrm{s}} \in$ $[12.5,15.5] \mathrm{mag}$, this method leads to the density map presented
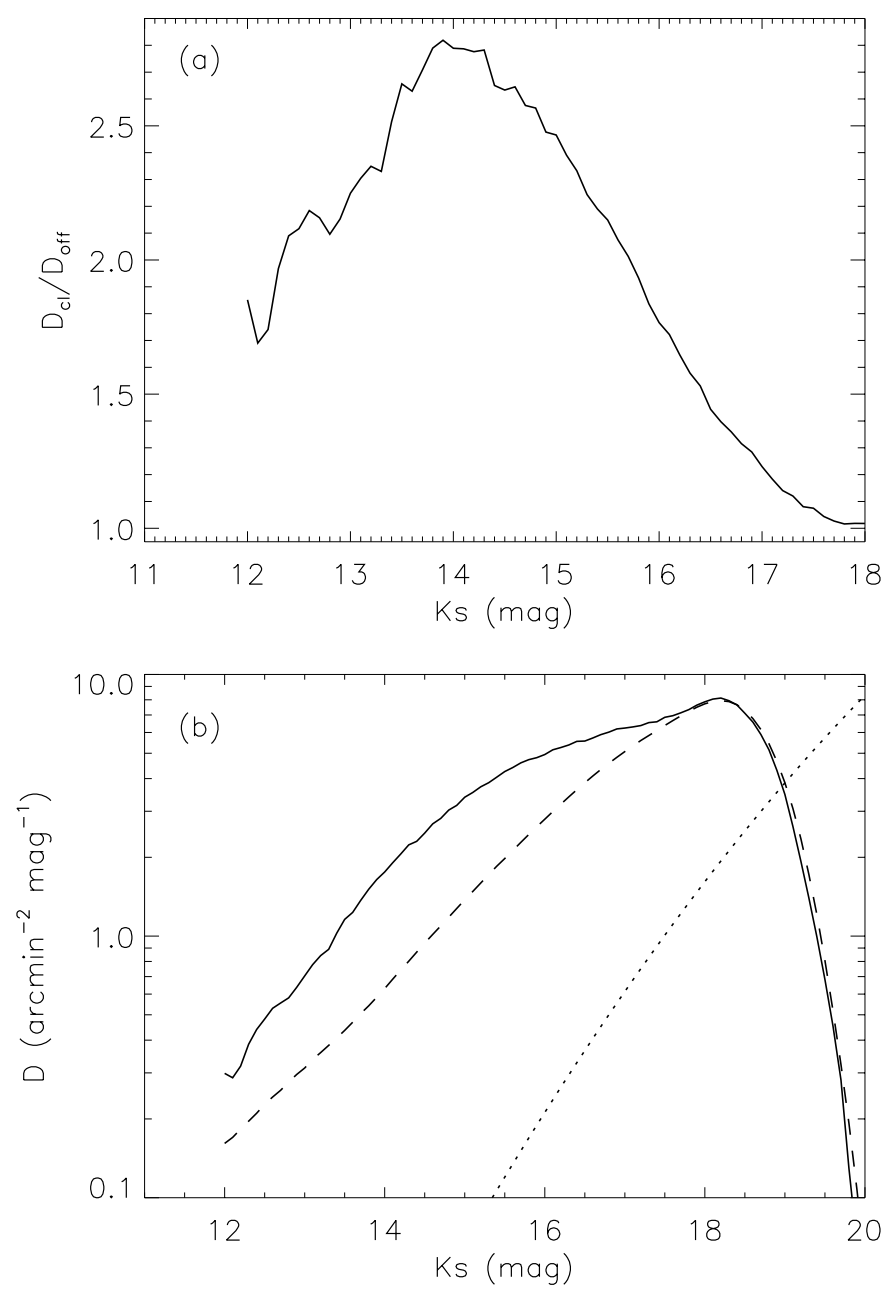

Fig. 4. Top: average stellar density ratio for $K_{\mathrm{s}} \in[m-1, m]$ between the cluster's on and off positions. Bottom: average stellar density for $K_{\mathrm{s}} \in$ $[m-1, m]$ for the cluster's on and off positions with solid and dashed lines, respectively. The dotted line represents the density of galaxies (Gardner et al. 1997).

in Fig. 5a. Its median stellar density is $3.1 \pm 0.5 \operatorname{arcmin}^{-2}$. We define clusters as a density threshold $3 \sigma$ above that level, i.e., above $4.6 \mathrm{arcmin}^{-2}$. The corresponding spatial resolution for the mapping is $1.1^{\prime}$ on the cluster edge, it is better inside since the stellar density is higher.

The source density (Fig. 5a) and the extinction maps (Fig. 2) share anticorrelated structures. This is the expected consequence of extinction that makes the stellar density decrease. The extinction structures, which trace the interstellar matter distribution, are background variations of the star surface density map. They need to be subtracted in order to apply a cluster detection algorithm on a flat density map. Once the extinction map has been built, these background variations are easily predictable and the corrected map is obtained as follows:

$D_{\text {corr }}=D \times 10^{a A_{K_{\mathrm{S}}}}$

where $A_{K_{\mathrm{s}}}=0.112 A_{V}, a=0.318$ is our measure of the slope of the $K_{\mathrm{s}}$ luminosity function, and $D$ and $D_{\text {corr }}$ are the observed and the true stellar densities, respectively. The true, absorptionfree, density map is presented in Fig. 5b. The molecular cloud structures are no longer visible. The background is flat and set to zero by subtracting the median value of the map. The result is a field-star-subtracted surface density map for which structures are true stellar overdensities. 

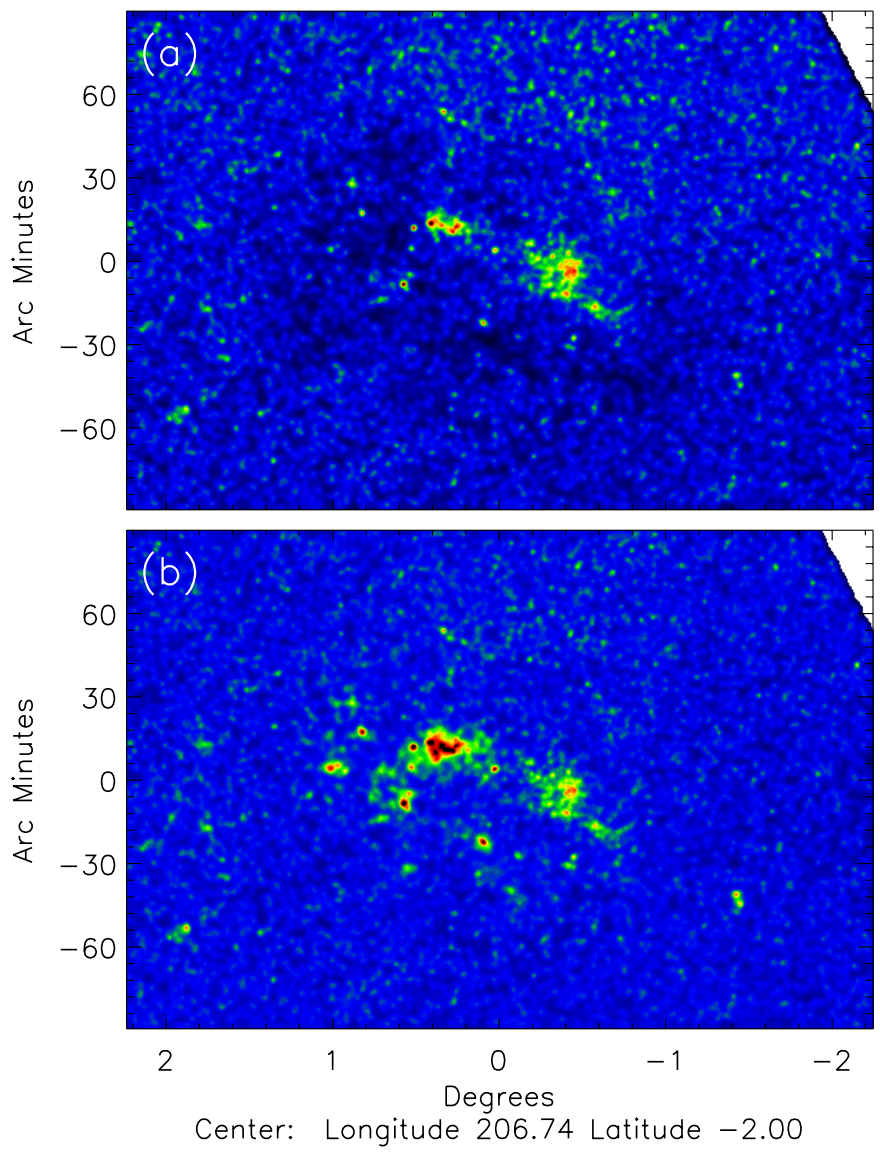

Fig. 5. Top: raw stellar density map. Bottom: stellar density map corrected for the interstellar extinction.

It is important to realize that all stars have been implicitly assumed to be background when using Eq. (3), whereas this is unknown for the cluster members themselves. Clusters can be either embedded or on the rear or the front side of the molecular cloud, and they will not suffer from the same amount of extinction depending on the case. Once the correction is done, setting the background to zero subtracts the field star density everywhere, including toward clusters. It is then possible to reverse the extinction correction on the clusters only assuming they would be on the front side rather than the rear side of the cloud. The final result for the overdensity map $D_{0}=D_{\text {corr }}-\bar{D}_{\text {corr }}$, with $\bar{D}_{\text {corr }}$ the median density, can be written as

$$
D_{0}=\underbrace{D_{\text {field }}^{L_{\text {corr }}} \times 10^{a A_{K_{\mathrm{s}}}}-\bar{D}_{\text {in }}}_{\text {noise, } \sigma=0.5 \operatorname{arcmin}^{-2}}+ \begin{cases}D^{\mathrm{cl}} \times 10^{a A_{K_{\mathrm{s}}}} & \text { back cluster } \\ D^{\mathrm{cl}} & \text { front cluster }\end{cases}
$$

where $D^{\text {field }}$ and $D^{\text {cl }}$ stand for field star and cluster densities, respectively. Both cases are taken into account when estimating the number of members per cluster in the next section.

\subsubsection{Cluster characterization}

We applied the clump detection algorithm clumpfind developed by Williams et al. (1994) on our density map. Its original 3D implementation for spectral line datacubes was generalized to $2 \mathrm{D}$ for submillimeter maps. Although clumpfind was designed to detect gas or dust clumps, it finds structures by connecting pixels of similar values, regardless of what the data represent. The detected structures in the star density map are stellar clusters by definition. In the clumpfind philosophy, each peak is a clump maximum. The peaks are selected above a level provided by the user, and the lowest level is the threshold for detection. Obviously the threshold definition would have been a serious issue with the raw density map with no extinction correction, since its background level is not uniform. We applied the IDL version of the clumpfind algorithm on the corrected density map (Fig. 5b) with the levels 3, 20, 25, and $40 \operatorname{arcmin}^{-2}$, meaning a cluster must have a star density excess greater than $3 \operatorname{arcmin}^{-2}$. Figure 6 shows the result with the clusters identification from Phelps \& Lada (1997) and Román-Zúñiga et al. (2008).

The levels 20, 25, and $40 \mathrm{arcmin}^{-2}$ are arbitrary chosen to split the connected clusters PL 04, PL 05, PL 06, and REFL 08 in accordance with Román-Zúñiga et al. (2008). As pointed out by Pineda et al. (2009), the clumpfind decomposition in crowded regions does not provide a robust list of physically meaningful clumps, although it is relevant for statistical analysis. We found 13 clusters (NGC 2244, NGC 2237, PL 01-07 from Phelps \& Lada 1997; REFL 08,09 from Román-Zúñiga et al. 2008) and two new clusters CMFT 10 and CMFT 11, which we named following the other cluster designation in this region using the author's initials; we do not confirm the existence of REFL 10

The total number of members for each cluster is obtained by integrating the density map over their surface area; however, the extinction correction applied with Eq. (3) on the whole map is only justified for clusters on the back of the molecular cloud and is irrelevant if they are located on the front side. As mentioned above, it is possible to compute the member numbers assuming cluster are on the front by reversing the correction in agreement with Eq. (4). The results presented in Table 1 provide the statistical number of members assuming the cluster is on the back or the front side of the cloud. The more the extinction towards a cluster, the greater the difference between these two numbers. The most affected cluster is REFL 08 with an estimated population that varies by a factor of 4 depending its location along the line of sight. The number of YSOs in Table 1 are for $K_{\mathrm{s}}<15.5 \mathrm{mag}$, which corresponds to $65 \%$ of the total star overdensity following Fig. 4. Therefore the total number of YSOs in the Rosette complex is expected to range between 4000 and 8000 members. We stress that this estimate misses only a small fraction of the total number of YSOs, which are the very young protostars (Class 0 ) that are undetectable at $K_{\mathrm{s}}$ because they emit mostly in the farinfrared and submillimeter wavelengths. For instance, the magnitude of a Class I source of $10^{6}$ years and only $0.1 M_{\odot}$ would be $K_{\mathrm{s}} \approx 15.5 \mathrm{mag}$ according to the model tool described in Siess et al. (2000). This is easily detected with UKIDSS, and it explains why the stellar surface density at $K_{\mathrm{s}}>17$ mag does not exhibit any more excess (see Fig. 4). Deeper observations at this wavelength would not improve YSO detection rate.

The number of YSOs permits the star formation efficiency (SFE) to be estimated per cluster. It is defined as the ratio of the YSO mass over the total mass (YSOs and gas). The YSO mass is derived from their number given in Table 1 for the back and the front cases (Cols. 6 and 7) and assuming a mean stellar mass of $0.6 M_{\odot}$. The gas mass is directly measured on the extinction map within each cluster boundary. Table 2 presents the results. For NGC 2244 and NGC 2237 the SFE is likely overestimated since these two clusters are located in the region where the gas has been swept up by the OB stars. For the others, the values range between 10 to $24 \%$ or 3 to $12 \%$ depending the assumed location of the clusters along the line of sight. This is to compare with the maximum efficiency of $\sim 30 \%$ derived from the mass function of dense molecular cores (Alves et al. 2007). 


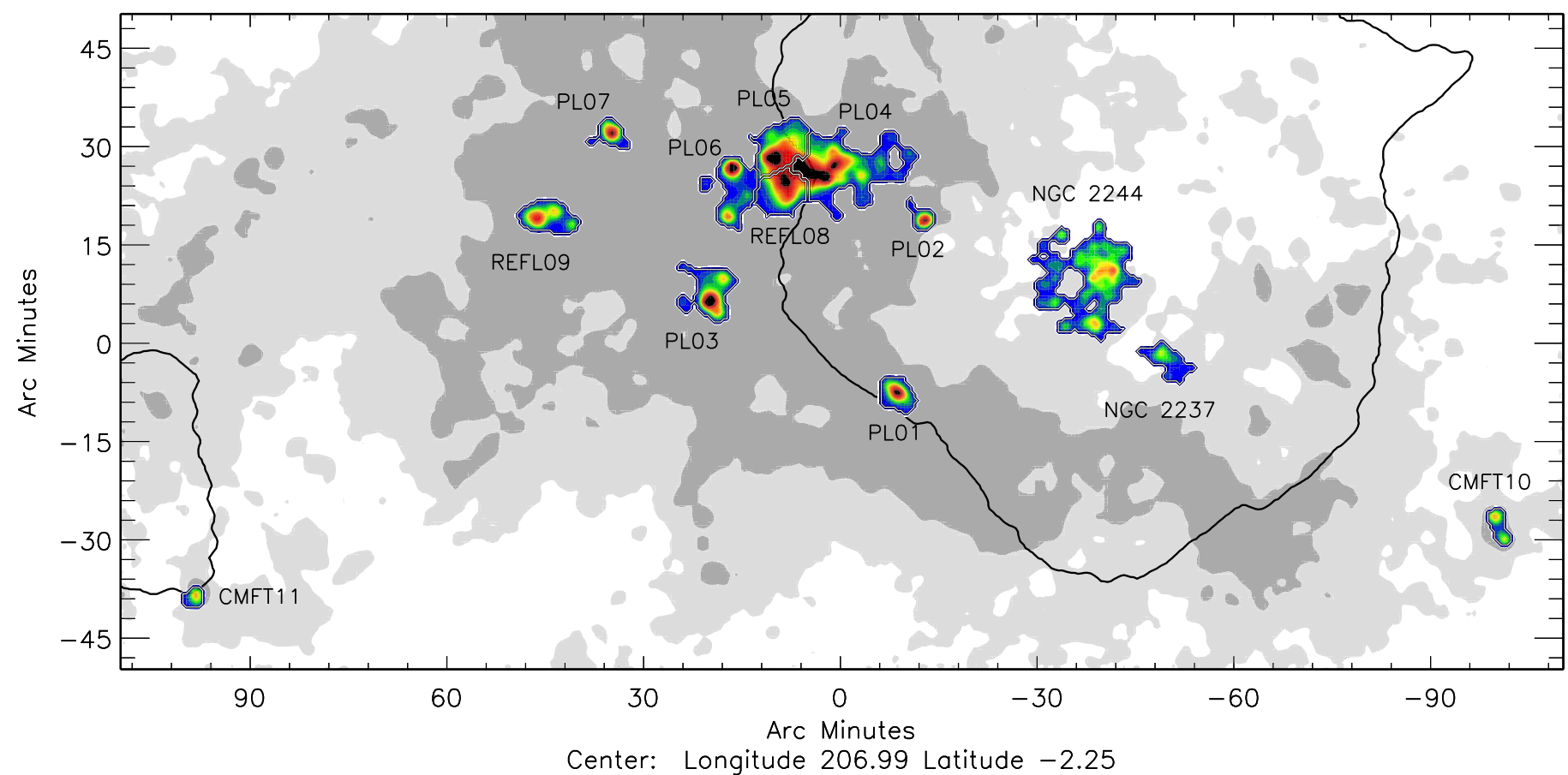

Fig. 6. Cluster detections in the Rosette molecular cloud. PL and REFL designations are from Phelps \& Lada (1997) and Román-Zúñiga et al. (2008), respectively. The solid black line shows the $\mathrm{H} \alpha$ contour that indicates the ionization front of the Rosette HII nebula centered on NGC 2244 and of Sh 2-280 to the east of the map. The two levels of shaded gray correspond to visual extinction of 2 and 4 mag, respectively. CMFT 10 and CMFT 11 are new clusters.

Table 1. Cluster characteristics.

\begin{tabular}{|c|c|c|c|c|c|c|c|c|c|c|c|c|}
\hline $\begin{array}{l}\text { Name } \\
(1) \\
\end{array}$ & $\begin{array}{c}\text { Glon Glat } \\
\text { deg } \\
(2) \\
\end{array}$ & $\begin{array}{l}\Delta \text { Glon } \\
\text { arc }\end{array}$ & $\Delta$ Glat & $\begin{array}{r}\text { Glon }^{\text {peak }} \\
\text { de } \\
(4)\end{array}$ & $\begin{array}{l}\text { Glat }^{\text {peak }} \\
\mathrm{g} \\
\end{array}$ & $\begin{array}{l}\operatorname{Max}^{\mathrm{B}} \\
\operatorname{arcn}\end{array}$ & $\begin{array}{l}\mathrm{Max}^{-2} \\
\mathrm{f}^{\mathrm{F}}\end{array}$ & $\begin{array}{l}N^{\mathrm{B}} \\
\text { (6) } \\
\end{array}$ & $\begin{array}{l}N^{\mathrm{F}} \\
(7) \\
\end{array}$ & $\begin{array}{c}N^{\text {cat }} \\
\text { UKIDSS } \\
(8) \\
\end{array}$ & $\begin{array}{c}\text { Area } \\
\operatorname{arcmin}^{2} \\
(9)\end{array}$ & $\begin{array}{c}\overline{A_{V}} \\
\mathrm{mag} \\
(10)\end{array}$ \\
\hline NGC 2244 & $206.368-2.096$ & 17.5 & 20.0 & 206.293 & -2.067 & 17.2 & 14.3 & 912 & 763 & 3840 & 169.7 & 2.2 \\
\hline NGC 2237 & $206.160-2.304$ & 8.8 & 7.1 & 206.176 & -2.275 & 9.6 & 7.9 & 132 & 106 & 668 & 29.7 & 2.6 \\
\hline PL01 & $206.839-2.387$ & 5.6 & 5.6 & 206.843 & -2.383 & 40.3 & 11.9 & 203 & 77 & 387 & 26.5 & 10.9 \\
\hline PL02 & $206.785-1.925$ & 4.3 & 4.6 & 206.777 & -1.942 & 29.8 & 9.5 & 102 & 46 & 195 & 13.5 & 8.5 \\
\hline PL03 & $207.327-2.125$ & 7.8 & 9.3 & 207.319 & -2.150 & 79.8 & 23.2 & 433 & 174 & 875 & 51.7 & 9.6 \\
\hline PL04 & $206.952-1.825$ & 20.1 & 10.5 & 207.027 & -1.833 & 53.9 & 17.0 & 1241 & 536 & 2461 & 136.7 & 8.6 \\
\hline PL05 & $207.143-1.758$ & 8.5 & 8.6 & 207.152 & -1.783 & 87.5 & 32.6 & 683 & 293 & 1096 & 55.7 & 9.2 \\
\hline REFL08 & $7.144-1.871$ & 7.7 & 7.8 & 207.127 & -1.842 & 39.4 & 5.5 & 533 & 125 & 473 & 46.0 & 16.0 \\
\hline PL06 & $7.273-1.875$ & 7.3 & 14.1 & 207.260 & -1.808 & 73.3 & 18.6 & 394 & 137 & 670 & 57.7 & 12.1 \\
\hline PL07 & $207.581-1.721$ & 5.5 & 4.7 & 207.569 & -1.716 & 28.7 & 9.3 & 140 & 53 & 238 & 19.2 & 10.8 \\
\hline REFL09 & $207.731-1.933$ & 10.4 & 5.5 & 207.760 & -1.941 & 20.8 & 5.3 & 265 & 88 & 463 & 39.7 & 12.7 \\
\hline CMFT1C & $205.313-2.720$ & 4.2 & 7.7 & 205.325 & -2.691 & 12.2 & 6. & 91 & 52 & 305 & 15.5 & 6.5 \\
\hline CMFT11 & $208.636-2.898$ & 3.8 & 3.8 & 208.628 & -2.890 & 12.4 & 6.4 & 60 & 38 & 212 & 10.2 & 5.0 \\
\hline
\end{tabular}

Notes. Column (2) contains the cluster geometrical centers followed by their dimensions. Column (4) is positions of the density peaks followed by two estimations for the peak value assuming a back or a front side location of the clusters. Columns (6) and (7) are the statistical numbers of members within clusters for back and front clusters; Col. (8) provides the total number of UKIDSS sources used to derive the extinction $\left(K_{\mathrm{s}}<17.8 \mathrm{mag}\right)$, and Col. (10) gives the average visual extinction for the cluster.

The observed SFE actually depends on the scale, from $10-30 \%$ for embedded clusters to only $1-5 \%$ for entire giant molecular clouds (Lada \& Lada 2003). This suggests that the RMC activity has not come to an end. PL 05 and CMFT 11 appear to be the most efficient at forming stars.

\section{Individual members}

This section focuses on the study of the 13 identified and well-delimited clusters to explore the role of triggering by the NGC 2244 OB stars in more detail. The UKIDSS deep nearinfrared data can be associated with the WISE observations at longer wavelengths to classify the sources located within the projected cluster boundaries. The classification process is complex because of the multiple contaminants, which are extragalactic sources, shock emission blobs, or resolved PAH emission structures. Koenig et al. (2012) propose a method of filtering all these sources and extracting YSOs. We started with their recommendations for building a first sample, and we improved completeness using a second method based on quadratic discriminant analysis. It is a statistical method for classification and pattern recognition in multidimensional data by looking for quadratic boundaries between groups of different object types. The training sample was prepared with the use of objects from previously 

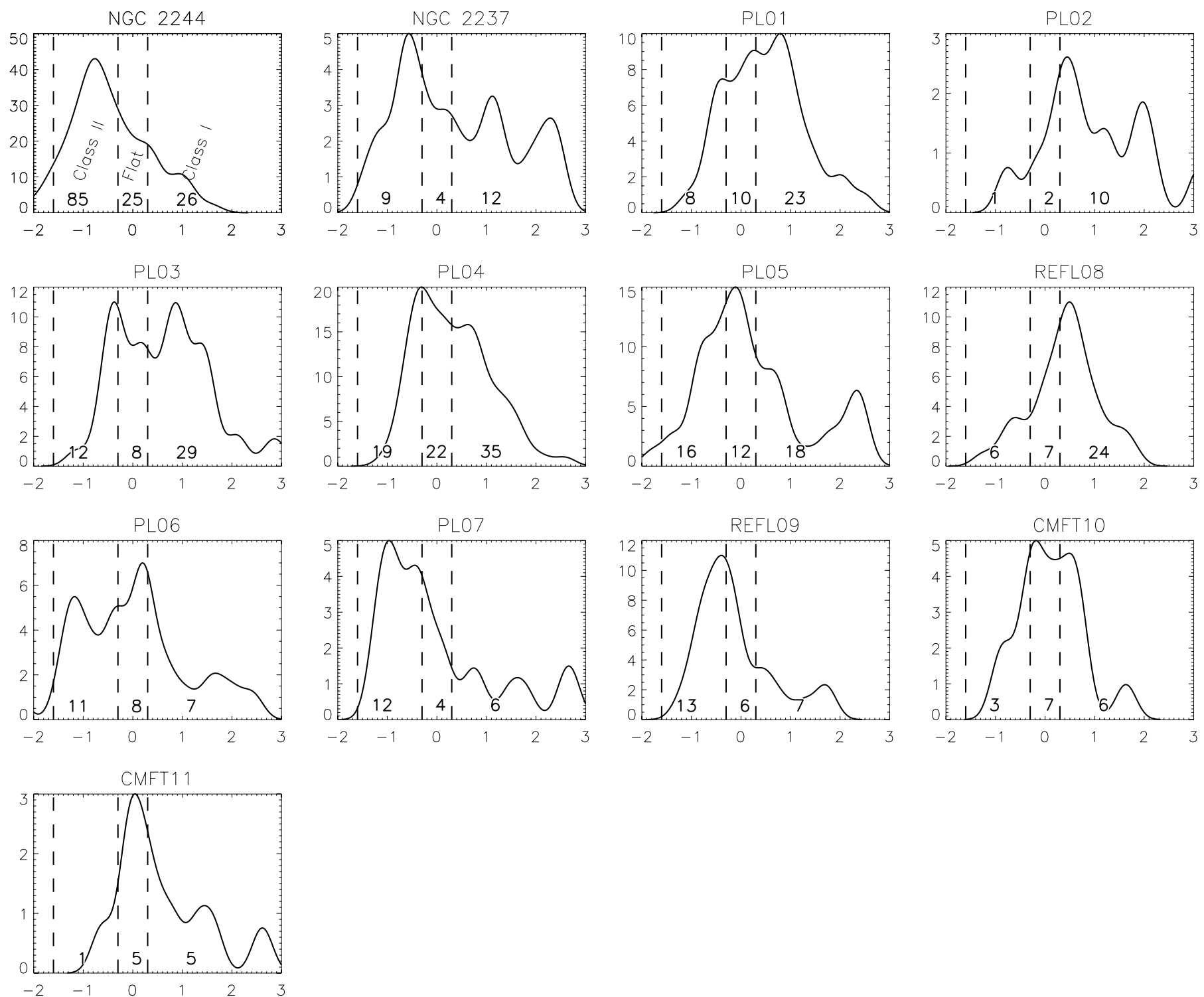

Fig. 7. Density distribution of the spectral index $\alpha=\operatorname{d} \log \left(\lambda F_{\lambda}\right) / \operatorname{dlog} \lambda$. The dashed lines define the Class II, Flat spectrum, and Class I source locations. The number of objects for these three classes is indicated for each cluster. The density distribution is obtained using the kernel estimator presented in Silverman (1986). It is particularly well adapted to small samples since it does not depend on the first bin position and is oversampled.

Table 2. Star formation efficiency for each cluster.

\begin{tabular}{lrcc}
\hline \hline Name & $\begin{array}{r}\text { Gas mass } \\
M_{\odot}\end{array}$ & $\begin{array}{c}\mathrm{SFE}^{\mathrm{B}} \\
\%\end{array}$ & $\begin{array}{c}\mathrm{SFE}^{\mathrm{F}} \\
\%\end{array}$ \\
\hline NGC 2244 & 1518 & 36.1 & 30.2 \\
NGC 2237 & 316 & 28.1 & 22.6 \\
PL01 & 1201 & 13.7 & 5.2 \\
PL02 & 469 & 17.0 & 7.6 \\
PL03 & 2075 & 16.4 & 6.6 \\
PL04 & 4834 & 19.4 & 8.4 \\
PL05 & 2049 & 23.8 & 10.2 \\
REFL08 & 3133 & 13.8 & 3.2 \\
PL06 & 2893 & 11.3 & 3.9 \\
PL07 & 901 & 12.7 & 4.8 \\
REFL09 & 2163 & 10.3 & 3.4 \\
CMFT10 & 472 & 15.3 & 8.7 \\
CMFT11 & 233 & 19.5 & 12.3 \\
\hline
\end{tabular}

Notes. Two SFE values are proposed to account for the uncertainty on the cluster locations with respect to the molecular cloud (the superscript $\mathrm{B}$ and $\mathrm{F}$ letters refer to the back and front side location as in Table 1). known types, i.e. galaxies, main-sequence stars, evolved stars, and YSOs, all listed in SIMBAD and VizieR. For a detailed description of the selection scheme see Marton et al. (in prep.).

We classified the YSOs according to the slope of the spectral index $\alpha=\mathrm{d} \log \left(\lambda F_{\lambda}\right) / \mathrm{d} \log \lambda$ (Greene et al. 1994). The source list of 535 YSO candidates with astrometry, photometry, and spectral index is published electronically at the CDS. Table 4 is a subsample that includes all candidates within the two new clusters CMFT 10 and CMFT 11. The spectral index is derived from a least square fitting from the $K_{\mathrm{s}}$ band to the third WISE channel, $W 3$, at $12 \mu \mathrm{m}$, whose passband actually extends from 8 to $18 \mu \mathrm{m}$. Sources are classified as Class I, flat spectrum, and Class II YSOs. Figure 7 shows the spectral index histogram for all clusters and the number of sources in each class. The spectral index depends mainly on the disk size and orientation, which implies its conversion into source age is not direct. However, it can be tested statistically with the spectral energy distribution (SED) fitting tool proposed by Robitaille et al. (2007), which provides the 10000 best possible solutions for a given energy distribution. We examined the correlation of $\alpha$ with the 1000 best fits for each 
Table 3. Age estimation of the cluster members detected by WISE based on their spectral index using Eq. (5).

\begin{tabular}{|c|c|c|c|c|}
\hline Name & Number & young & $\begin{array}{c}\text { Age (yr) } \\
\text { median }\end{array}$ & old \\
\hline NGC 2244 & 145 & $3.6 \times 10^{4}$ & $3.8 \times 10^{5}$ & $1.8 \times 10^{6}$ \\
\hline NGC 2237 & 25 & $1.1 \times 10^{4}$ & $8.7 \times 10^{4}$ & $1.0 \times 10^{6}$ \\
\hline PL01 & 41 & $1.5 \times 10^{4}$ & $5.8 \times 10^{4}$ & $2.9 \times 10^{5}$ \\
\hline PL02 & 13 & $1.1 \times 10^{4}$ & $3.3 \times 10^{4}$ & $2.0 \times 10^{5}$ \\
\hline PL03 & 49 & $1.1 \times 10^{4}$ & $3.3 \times 10^{4}$ & $2.7 \times 10^{5}$ \\
\hline PL04 & 76 & $1.6 \times 10^{4}$ & $8.0 \times 10^{4}$ & $3.2 \times 10^{5}$ \\
\hline PL05 & 47 & $1.1 \times 10^{4}$ & $1.2 \times 10^{5}$ & $6.1 \times 10^{5}$ \\
\hline REFL08 & 37 & $1.8 \times 10^{4}$ & $5.2 \times 10^{4}$ & $3.7 \times 10^{5}$ \\
\hline PL06 & 27 & $1.3 \times 10^{4}$ & $1.1 \times 10^{5}$ & $1.5 \times 10^{6}$ \\
\hline PL07 & 22 & $1.2 \times 10^{4}$ & $2.4 \times 10^{5}$ & $1.1 \times 10^{6}$ \\
\hline REFL09 & 26 & $1.9 \times 10^{4}$ & $2.1 \times 10^{5}$ & $5.8 \times 10^{5}$ \\
\hline CMFT10 & 16 & $3.4 \times 10^{4}$ & $9.4 \times 10^{4}$ & $5.2 \times 10^{5}$ \\
\hline CMFT11 & 11 & $1.2 \times 10^{4}$ & $7.2 \times 10^{4}$ & $2.1 \times 10^{5}$ \\
\hline
\end{tabular}

Notes. The columns labeled young and old contain the age estimate for the youngest and the oldest populations within each cluster. young and old refer to the $10 \%$ and $90 \%$ quantile, respectively.

star in two clusters, PL 07 and CMFT 11, which allow probing of a wide range of values. While $\alpha$ is measured only with the four fluxes from $K_{\mathrm{s}}$ to $W 3$, the SED fitting is performed on the seven fluxes from $J$ to $W 4$ to better constrain the source physical properties. The correlation between $\alpha$ and several parameters, such as the age, the central source mass, the disk inclination, the accretion rate, or the extinction, is analyzed by measuring the histogram peak of the 1000 best fits for each of these parameters. The only parameters that exhibits a clear correlation (coefficient of 0.9) with the spectral index is the age that follows the powerlaw

$\log \left(\right.$ Age $\left.-10^{4}\right)=(-0.88 \pm 0.07) \alpha+(5.02 \pm 0.08)$

Since the age provided by the Robitaille et al. (2007) model starts with the theoretical evolutionary tracks; i.e., after the protostar collapse, we arbitrarily added $10^{4}$ years to refer more specifically to an earlier stage. The age obtained from the fitting model is actually indirectly determined through constraining the circumstellar parameters and is used to give a rough idea of the stellar properties as a function of mass (T. Robitaille, priv. comm.). It cannot be considered as a reliable age measurement for individual stars, so we carefully limit its usage to a statistical analysis. Under this restriction, it is possible to interpret Fig. 7 as an age distribution. This histogram representation is a valuable tool that provides us with an immediate and comprehensive picture of the age distribution within a cluster. Table 3 provides the median age estimate for each cluster, together with the $10 \%$ and $90 \%$ quantiles of the sample. Quantiles are more robust to statistical fluctuations and yield an estimation of the cluster ages, which we believe is correct within a factor of 2 . The $10 \%$ quantile represents the age of the young population of the clusters, whereas the $90 \%$ quantile provides us with the age of the oldest population and therefore indicates the age of the beginning of the star formation in each cluster.

The oldest cluster is NGC 2244, which starts forming stars about $2 \mathrm{Myr}$, in agreement with the usual age determination of its $\mathrm{O}$ star population. Then the clusters tend to be younger as the distance to NGC 2244 increases. This is true for NGC 2237 , PL 01, PL 02. The youth of PL 01 and PL 02 would be consistent with a scenario where compression effects can indeed locally provoke the formation of clusters in the immediate interaction zone between the HII-region and molecular cloud (on a parsec scale of a few). Schneider et al. (2012) arrive at the same conclusion based on the large number of dense cores found in this interaction region. The age of PL 03 is similar, but it is located beyond the influence of NGC 2244. PL 05 is even older than these potentially triggered clusters, with about $6.1 \times 10^{5} \mathrm{yr}$. Going farther, PL 06 and PL 07 are older at more than 1 Myr and may even have formed at the same time as NGC 2244. Schneider et al. (2010) and Hennemann et al. (2010) argue that PL 07 would be the youngest cluster in the RMC, but their age classification was determined in a preliminary way and with lower quality statistics. We agree that there are young protostars in this cluster, but the relevant age that determines the beginning of the collapse is the one of the oldest population of the cluster, not the youngest. The collapse of the more distant clusters, REFL 09, CMFT 10, and CMFT 11, are similar to PL 04 whereas they are definitely out of reach of the OB stars in NGC 2244. We cannot rule out the possibility that some cluster formation has been triggered by NGC 2244, but it cannot be true for those at greater distances that are within the same age range. The overall picture favors the Heyer et al. (2006) claim also supported by Schneider et al. (2012) of a dynamical evolution of the whole cloud with a very minor triggering effect, if any.

Regarding the youngest population of the clusters, there is no evidence that any of them has stopped forming stars. This is confirmed by Herschel observations that discovered protostars and protostellar clumps (Hennemann et al. 2010; di Francesco et al. 2010) in all the clusters they have mapped. NGC 2244, REFL 09, and CMFT 10 would be more likely to be about to stop their activity. That is expected for NGC 2244 at the center of the HII region because most of its surrounding interstellar material has been swept up. The fraction of Class I sources should bring additional evidence. In Sect. 4 we have estimated the total number of members in clusters with $K_{\mathrm{s}}<15.5 \mathrm{mag}$. At this luminosity level, all Class I sources are easily detected in the four WISE channels since they have a positive spectral index, making them significantly brighter than the completeness limit. For instance, the expected WISE magnitudes for an object with $K_{\mathrm{s}}=15.5 \mathrm{mag}$ and a spectral index of 0 are $14.2,13.2,10.3$, and $8.2 \mathrm{mag}$. It is therefore meaningful to derive the fraction of Class I objects by comparing the total number of members from Table 1 with the number of Class I indicated in Fig. 7. The smallest fraction is obtained for NGC 2244 with 3\%, confirming that the activity of this cluster has severely declined. The highest fraction of Class I objects is found in PL 01 and PL 02 with $10-30 \%$ and $10-20 \%$, respectively. The wide range results from the uncertainty on the amount of extinction between the clusters and us. This high fraction does not prove triggering has occurred, but it certainly indicates that the OB star influence is not inhibiting the star formation in these two clusters, although their projected distance to NGC 2244 is only about 14 pc.

Our results need to be compared with other regions where the question of the influence of the massive stars on the surrounding interstellar medium is raised. For instance, Roccatagliata et al. (2013) analyzed Herschel data in the Carina nebula complex and claim that triggered star formation by radiative cloud compression is observed. Deharveng et al. (2005) searched for star forming regions triggered by a collect and collapse process that corresponds to the expansion of the HII nebula. They confirm the role of this process for regions such as RCW 79 and Sh2-212 in companion papers (Zavagno et al. 2006; Deharveng et al. 2008). Regarding the RMC we do not exclude that this process has contributed in the close interaction zone which includes NGC 2237 , 
Table 4. YSO candidates in the Rosette molecular cloud.

\begin{tabular}{|c|c|c|c|c|c|c|c|c|c|c|c|}
\hline WISE & GLON & GLAT & Cluster & $\alpha$ & $J$ & $H$ & $K_{\mathrm{s}}$ & W1 & W2 & W3 & W4 \\
\hline 1.6 & 3 & -1 & 0 & 0.2 & & 6 & 0 & 4 & 5 & 62 & 6.38 \\
\hline 07.5 & 0 & 1 & ИF & 0.1 & 186 & & & & & 8.72 & 51 \\
\hline 7 & 0 & 3 & MFT10 & 0.7 & 1684 & & & & & 83 & 3.31 \\
\hline 025.5 & 06 & 04 & CMFT10 & 1.6 & & & 17.50 & 4.82 & 3.78 & 9.14 & 36 \\
\hline 105.5 & 03 & .7468 & CMFT10 & 0.6 & 14.50 & .59 & 11.02 & 9.25 & 7.99 & 4.70 & 36 \\
\hline & & 2.65 & CMFT10 & -0.8 & 15.88 & 14.62 & 13.69 & 12.65 & 11.95 & 10.01 & 8.09 \\
\hline & & 2.7 & 10 & 0.5 & 13.23 & 12.78 & 12.42 & 3 & 11.27 & 6.52 & 4.64 \\
\hline & & -2.6 & $\mathrm{CMl}$ & & & & & & & & 5.80 \\
\hline & & -2.7 & & -0.2 & & & & & & 9.49 & 7.05 \\
\hline & & -2.7 & 10 & -0.9 & 16.01 & & & & 12.59 & 10.47 & 7.25 \\
\hline & & -2.746 & & 0.4 & & & 13.52 & & 10.77 & 7.51 & 5.62 \\
\hline & & -2.7 & & -0.5 & & & 13.48 & & 12.05 & 9.27 & 7.08 \\
\hline & & -2.6 & & -0.2 & & & & & 9.99 & 7.77 & 5.70 \\
\hline & & & & 0.7 & & & & & 12.43 & 9.30 & 6.61 \\
\hline & & ? & 10 & -0.3 & & & & & 8 & 8.48 & 5.55 \\
\hline & & & & -0 & & & & & 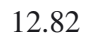 & & 6.33 \\
\hline & & 20 & 11 & -0 . & 16.06 & 14.62 & & 12 & 11.54 & 8.63 & 5.58 \\
\hline & & -2.8 & 11 & 2.6 & & & 38 & 11 & 7.8 & 5.78 & 0.99 \\
\hline & & 88 & 11 & 1. & 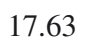 & 0 & 13.32 & & . & 5.80 & 2.95 \\
\hline & 208. & -2.8 & CMFT11 & 1. & & & & & 11 & 8.09 & 5.13 \\
\hline & & -2.8 & & 0 . & & & & & 9.98 & 7.55 & 4.57 \\
\hline & 208.6 & -2.903 & CMFT11 & 0.2 & 17. & 15.16 & & & 10.24 & 7.79 & 4.85 \\
\hline & 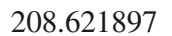 & -2.89 & CMFT11 & -0.0 & 16. & & & & 12.84 & 9.65 & \\
\hline & 208. & -2.8936 & CMFT11 & -0.6 & 14.38 & & 12.90 & & 11.07 & 8.80 & \\
\hline & 208.6 & -2.872707 & CMFT11 & 0.8 & & 18.46 & 16.62 & & 12.56 & 9.79 & 5.90 \\
\hline & 208.6 & -2.8868 & CMFT11 & 0.3 & & 17.20 & 15.14 & & 12.33 & 9.59 & 5.90 \\
\hline J063316.22+023037.2 & 208.622069 & -2.890993 & CMFT11 & -0.1 & 17.43 & 15.26 & 13.92 & 12.64 & 10.92 & 9.00 & 4.72 \\
\hline
\end{tabular}

Notes. The full table of 535 objects within 13 clusters is published electronically at the CDS, this subsample lists only the candidates for the two new clusters CMFT 10 and CMFT 11 . The astrometry and $W[1-4]$ photometry is from WISE; the $J H K_{\mathrm{s}}$ is from UKIDSS or 2MASS when $<12$ mag; $\alpha$ is the spectral index measured from $K_{\mathrm{s}}$ to $W 3$.

PL 01, and PL 02. Dewangan \& Ojha (2013) studied another mid-infrared bubble, [CPA2006] N14, and concluded that this effect was not consistent with the dynamical time scales and suggest that a compression by a shock wave is more likely to have triggered the star formation. Tackenberg et al. (2013) studied the star formation in the infrared dark cloud SDC G18.928-0.031 associated with an HII region. Despite the morphology of the complex, which suggests triggered star formation, they did not find evidence that the massive clump is prone to collapse because of the expanding HII region. Although the general characteristics of these complexes, which associate a molecular cloud with an HII region, look similar, their evolution varies. This is likely the result of the 3D structures, which are generally not known. In a projected image we may not always be sure which clump is exposed to the increased radiation energy density with UV photons. This is supported by the theoretical works of Dale \& Bonnell $(2011,2012)$, which show that star formation may occur in dense filaments protected from ionizing sources, with preexisting bubbles shaped by turbulence that mimics a triggered star forming region geometry.

\section{Conclusion}

We performed a large scale study of the Rosette complex with near and mid-infrared data, which allowed us to map the extinction and the young star cluster surface densities. We proposed a new method based on the joint analysis of star color and density to identify embedded star clusters and to derive the main characteristics of their YSO population. In particular, we showed that extinction mapping is essential for removing the fluctuations of the field star surface density resulting from the interstellar medium environment. Extinction is also critical to estimating the number of stars in excess, i.e. YSOs, within a molecular cloud. We found 13 clusters in the RMC, two of them new discoveries. The total number of YSOs within these clusters is between 4000 and 8000 stars depending on the cluster locations along the line of sight with respect to the parent molecular cloud. The luminosity function of the YSOs peaks at $K_{\mathrm{s}} \approx 14 \mathrm{mag}$, and very few objects are beyond $17 \mathrm{mag}$ at $K_{\mathrm{s}}$, which points out that UKIDSS is sensitive enough to reach completeness in this region.

We identified 535 individual members within the clusters using WISE photometry. The analysis of their SED from 1 to $22 \mu \mathrm{m}$ allowed us to estimate the age and the current status of each cluster. We concluded that they all have recent activity and that a triggering scenario for the star formation in the Rosette is not consistent with all the observed cluster ages. Despite the presence of $\mathrm{OB}$ stars in the Rosette nebula center, no evidence of triggering or inhibiting star formation has been found except for NGC 2244.

More generally, the method we have developed to investigate embedded clusters can be applied to other active regions to better constrain the impact of triggering in star formation. This process is mostly invoked on a simple geometrical basis with little physical analysis since it provides a plausible explanation of the presence of clusters close to an $\mathrm{OB}$ association. We suggest that this assumption is actually too naive and that a deeper analysis, such as this one, is required to confirm the actual role of massive stars on the surrounding star formation.

Acknowledgements. L. Cambrésy wish to thank O. Roos for her contribution in initiating the project. G. Marton has been supported by the Hungarian Research Fund (OTKA K-104607) and by the PECS program of the European Space Agency and the Hungarian Space Office (PECS-98073). This research 
was funded partly by the grant OTKA 101393. This work is based in part on data obtained as part of the UKIRT Infrared Deep Sky Survey. This publication makes use of data products from the Two Micron All Sky Survey, which is a joint project of the University of Massachusetts and the Infrared Processing and Analysis Center/California Institute of Technology, funded by the National Aeronautics and Space Administration and the National Science Foundation. This publication makes use of data products from the Wide-field Infrared Survey Explorer, which is a joint project of the University of California, Los Angeles, and the Jet Propulsion Laboratory/California Institute of Technology, funded by the National Aeronautics and Space Administration.

\section{References}

Alves, J., Lombardi, M., \& Lada, C. J. 2007, A\&A, 462, L17

Balog, Z., Muzerolle, J., Rieke, G. H., et al. 2007, ApJ, 660, 1532

Cambrésy, L., Beichman, C. A., Jarrett, T. H., \& Cutri, R. M. 2002, AJ, 123, 2559

Cambrésy, L., Rho, J., Marshall, D. J., \& Reach, W. T. 2011, A\&A, 527, A141

Carpenter, J. M. 2000, AJ, 120, 3139

Casali, M., Adamson, A., Alves de Oliveira, C., et al. 2007, A\&A, 467, 777

Casertano, S., \& Hut, P. 1985, ApJ, 298, 80

Celnik, W. E. 1985, A\&A, 144, 171

Dale, J. E., \& Bonnell, I. 2011, MNRAS, 414, 321

Dale, J. E., \& Bonnell, I. A. 2012, MNRAS, 422, 1352

Deharveng, L., Zavagno, A., \& Caplan, J. 2005, A\&A, 433, 565

Deharveng, L., Lefloch, B., Kurtz, S., et al. 2008, A\&A, 482, 585

Dewangan, L. K., \& Ojha, D. K. 2013, MNRAS, 429, 1386

di Francesco, J., Sadavoy, S., Motte, F., et al. 2010, A\&A, 518, L91

Elmegreen, B. G. 1998, in Origins, eds. C. E. Woodward, J. M. Shull, \& H. A. Thronson, Jr., ASP Conf. Ser., 148, 150

Gardner, J. P., Sharples, R. M., Frenk, C., \& Carrasco, B. 1997, ApJ, 480, L99

Greene, T. P., Wilking, B. A., Andre, P., Young, E. T., \& Lada, C. J. 1994, ApJ, 434,614

Hambly, N. C., Collins, R. S., Cross, N. J. G., et al. 2008, MNRAS, 384, 637

Hennemann, M., Motte, F., Bontemps, S., et al. 2010, A\&A, 518, L84

Hewett, P. C., Warren, S. J., Leggett, S. K., \& Hodgkin, S. T. 2006, MNRAS, 367,454
Heyer, M. H., Williams, J. P., \& Brunt, C. M. 2006, ApJ, 643, 956

Hodgkin, S. T., Irwin, M. J., Hewett, P. C., \& Warren, S. J. 2009, MNRAS, 394 675

Kainulainen, J., Alves, J., Beuther, H., Henning, T., \& Schuller, F. 2011, A\&A, 536, A48

Koenig, X. P., Leisawitz, D. T., Benford, D. J., et al. 2012, ApJ, 744, 130

Lada, C. J., \& Lada, E. A. 2003, ARA\&A, 41, 57

Lawrence, A., Warren, S. J., Almaini, O., et al. 2007, MNRAS, 379, 1599

Motte, F., Zavagno, A., Bontemps, S., et al. 2010, A\&A, 518, L77

Phelps, R. L., \& Lada, E. A. 1997, ApJ, 477, 176

Pineda, J. E., Rosolowsky, E. W., \& Goodman, A. A. 2009, ApJ, 699, L134

Poulton, C. J., Robitaille, T. P., Greaves, J. S., et al. 2008, MNRAS, 384, 1249

Rieke, G. H., \& Lebofsky, M. J. 1985, ApJ, 288, 618

Robitaille, T. P., Whitney, B. A., Indebetouw, R., \& Wood, K. 2007, ApJS, 169, 328

Roccatagliata, V., Preibisch, T., Ratzka, T., \& Gaczkowski, B. 2013, A\&A, 554, A6

Román-Zúñiga, C. G., \& Lada, E. A. 2008, in Star Formation in the Rosette Complex (ASP Monograph), 928

Román-Zúñiga, C. G., Elston, R., Ferreira, B., \& Lada, E. A. 2008, ApJ, 672, 861

Roy, A., Martin, P. G., Polychroni, D., et al. 2013, ApJ, 763, 55

Russeil, D., Schneider, N., Anderson, L. D., et al. 2013, A\&A, 554, A42

Savage, B. D., \& Mathis, J. S. 1979, ARA\&A, 17, 73

Schneider, N., Motte, F., Bontemps, S., Hennemann, M., et al. 2010, A\&A, 518, L83

Schneider, N., Csengeri, T., Hennemann, M., et al. 2012, A\&A, 540, L11

Siess, L., Dufour, E., \& Forestini, M. 2000, A\&A, 358, 593

Silverman, B. 1986, Density Estimation for Statistics and Data Analysis (Taylor \& Francis)

Skrutskie, M. F., Cutri, R. M., Stiening, R., et al. 2006, AJ, 131, 1163

Tackenberg, J., Beuther, H., Plume, R., et al. 2013, A\&A, 550, A116

Wang, J., Feigelson, E. D., Townsley, L. K., et al. 2009, ApJ, 696, 47

Williams, J. P., de Geus, E. J., \& Blitz, L. 1994, ApJ, 428, 693

Williams, J. P., Blitz, L., \& Stark, A. A. 1995, ApJ, 451, 252

Wright, E. L., Eisenhardt, P. R. M., Mainzer, A. K., et al. 2010, AJ, 140, 1868

Zavagno, A., Deharveng, L., Comerón, F., et al. 2006, A\&A, 446, 171

Pages 11 to 12 are available in the electronic edition of the journal at http://wWw . aanda. org 


\section{Appendix A: Comparison with the column density obtained from Herschel data}

The extinction map derived in this work relies on the reddening of background sources detected at $H$ and $K_{\mathrm{s}}$ by UKIDSS. A totally independent estimation of the column density is proposed by Schneider et al. (2012) from dust emission. Their map is based on the gray-body fitting from 160 to $500 \mu \mathrm{m}$ of the $1.8 \mathrm{deg}^{2}$ observed by the Herschel key program HOBYS (Motte et al. 2010). Using our extinction map as reference, we convert the Herschel column density (noted $C D$ and expressed in $\mathrm{cm}^{-2}$ ) into extinction as $A_{V}(\mathrm{FIR})=\left(C D-3.71 \times 10^{20}\right) / 5.11 \times 10^{20}$. This relation depends mainly on the dust opacity at all wavelengths, and the gas-to-dust ratio through the value of $N_{\mathrm{H}} / A_{V}$ that is supposed constant. However, the situation is more complicated, as pointed out by a recent study by Roy et al. (2013), which discusses the variation in the dust opacity with column density in Orion. They conclude that no single opacity can be applied to the whole cloud, which has quantitative implications for interpreting of the column density from Herschel data. Differences between the two column density maps are therefore expected.

Figure A.1 presents the difference between the two extinctions as a function of the distance to NGC 2244, i.e. the OB stars. The excess of extinction for the region at less than $\sim 50^{\prime}$ from the NGC 2244 suggests that the optical depth derived from dust emission is overestimated near the massive star cluster. The spatial distribution of the excess is shown better in Fig. A.2, which presents the two extinction maps and their difference. The diffuse excess, which appears in green in Fig. A.2c recalls the ionization front of Fig. 6. The extinction excess likely results from the emission of warmer grains in the diffuse envelop surrounding the dense molecular cloud. A similar effect has been observed by Russeil et al. (2013) in NGC 6334 when excluding the $70 \mu \mathrm{m}$ to derive the column density. In addition, the two maps exhibit strong differences of several tens of magnitudes for limited areas that happen to match the star clusters. It suggests local heating by YSOs, although it is true that real dense cores with $A_{V}>150$ mag would not be revealed using $H-K_{\mathrm{s}}$ since no background star could be detected through such a column density.

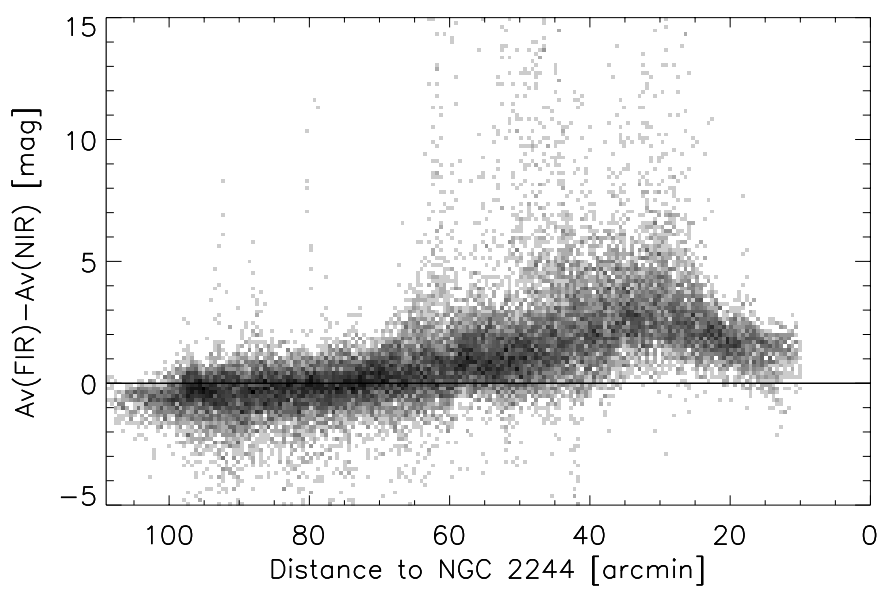

Fig. A.1. Difference between the extinction obtained from dust emission in the FIR and from dust reddening in the NIR versus the distance to NGC 2244. The $\mathrm{x}$-axis corresponds roughly to the galactic longitude and is reversed to match the map orientation with east pointing left.

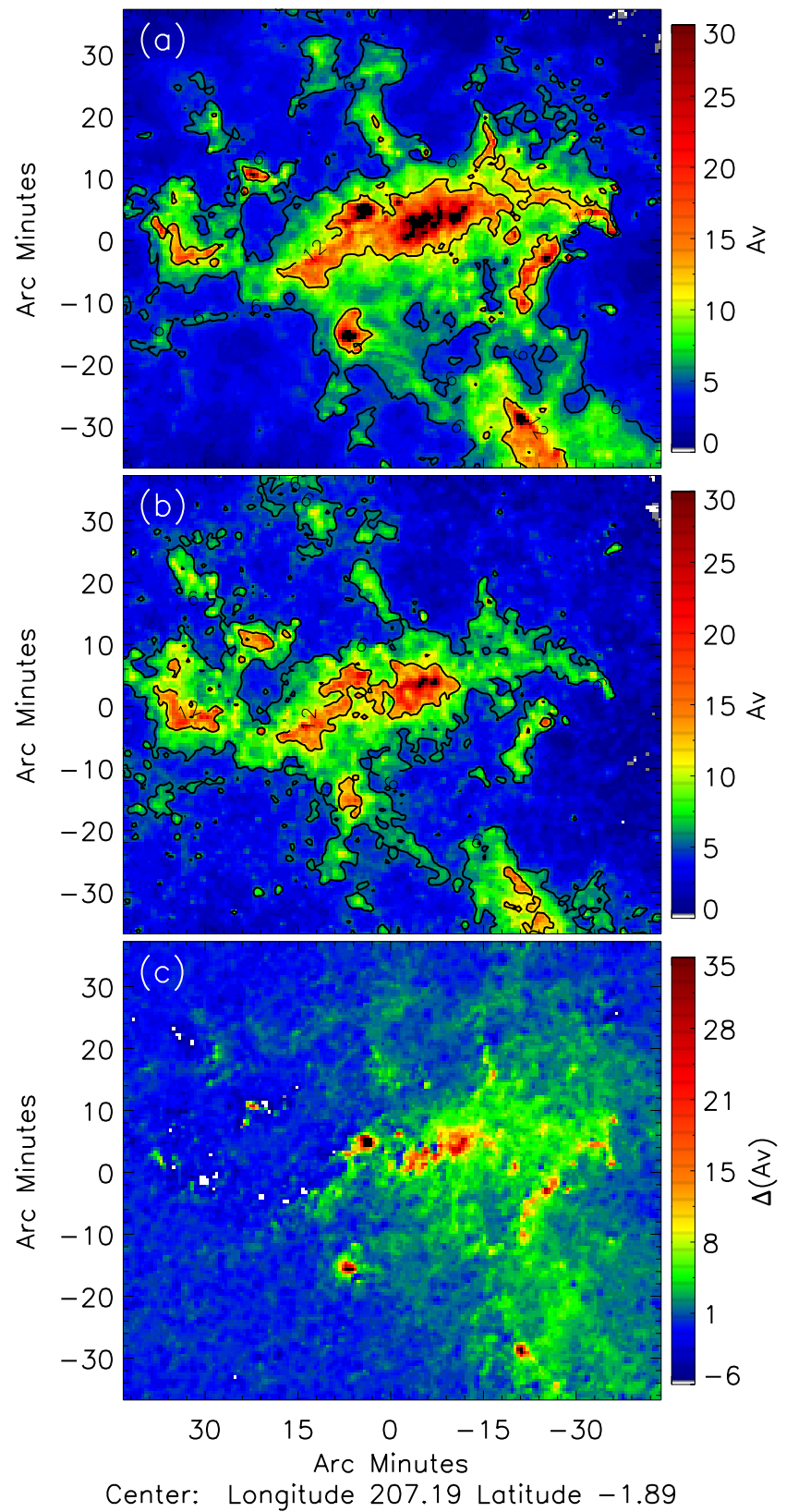

Fig. A.2. a) Extinction derived from dust emission using Herschel data. b) Extinction derived from UKIDSS $H-K_{\mathrm{s}}$ color excess. c) Difference between the two extinction map a)-b). The color-map cuts are identical for a) and b) and set to display the visual extinction from 0 to 30 mag. Contours for $A_{V}=6$ and $12 \mathrm{mag}$ are overlaid.

Despite the obvious differences between the two extinction maps, it is worth noting the total mass estimates shown in Fig. A.3 are consistent. We obtained $1.7 \times 10^{5}$ and $1.4 \times 10^{5} M_{\odot}$ for the Herschel and the UKIDSS-based maps, respectively. The high extinctions make only a very limited contribution to the total mass estimate because they correspond to a small surface area. Our analysis for the RMC confirms that Herschel column density maps must be used with caution. Further comparisons between column density maps derived with Herschel and extinction methods are required to better understand the impact of parameters, such as the opacity (i.e. dust emissivity), variations, and the presence of several dust components along the line of sight. 
A\&A 557, A29 (2013)

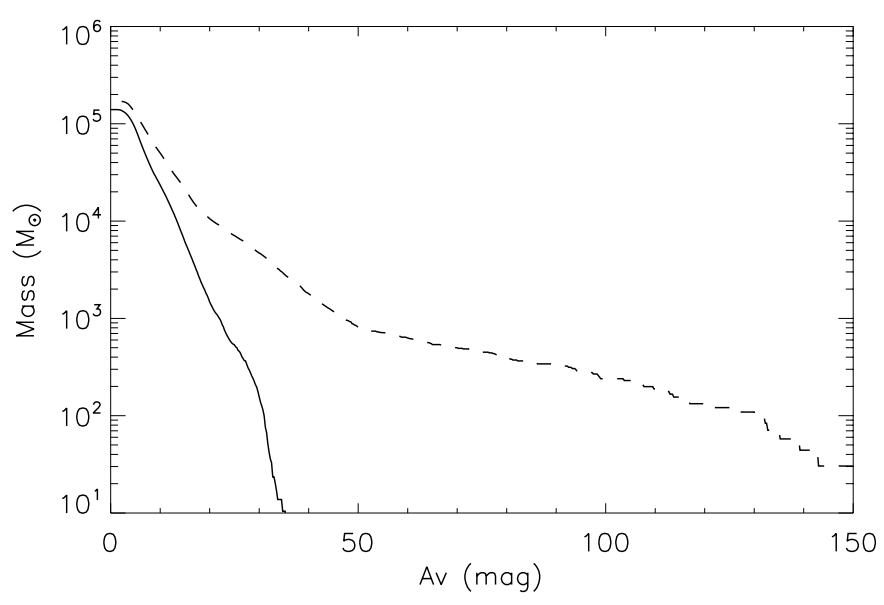

Fig. A.3. Cumulative mass distribution from Herschel dust emission (dashed line) and UKIDSS reddening (plain line) for the same field of view. 